Acta Crystallographica Section D

Biological

Crystallography

ISSN 0907-4449

Vincenzo Granata, ${ }^{a}$ Nicholas G. Housden, ${ }^{\mathrm{b}}$ Stephen Harrison, ${ }^{\mathrm{b}}$ Colette Jolivet-Reynaud, Michael G. Gore ${ }^{\mathrm{b}}$ and Enrico Adriano Stura ${ }^{d}$ *

${ }^{a}$ Università Federico II, Dipartimento di Chimica Biologica, Via Mezzocannone, Napoli, Italy, ${ }^{\mathbf{b}}$ Institute of Biomolecular Sciences, University of Southampton, Bassett Crescent East, Southampton SO16 7PX, UK, ' Department of R\&D Immunoassays, BioMérieux, Marcy I'Étoile, France, and d Départment d'Ingénierie et d'Etudes des Protéines (DIEP), C.E. Saclay, 91191 Gif-sur-Yvette, France

Correspondence e-mail: estura@cea.fr

Received 18 September 2004

Accepted 7 March 2005

\section{Comparison of the crystallization and crystal packing of two Fab single-site mutant protein $\mathrm{L}$ complexes}

Protein L from Peptostreptococcus magnus ( $\mathrm{PpL}$ ) is a multidomain protein composed of four or five immunoglobulin-binding domains that target the $\kappa$ light chain of a large repertoire of human and murine antibodies. Thus, a single domain of this protein can be used to aid the crystallization of Fab, free or complexed to their antigen when it is not possible to obtain crystals without it. Each wild-type PpL domain has two light-chain binding sites that target the same region of the light chain and can thus bring together two Fab-antigen complexes within the crystal lattice. In this context the small $\mathrm{PpL}$ domain is sandwiched between two Fab and cannot participate in crystal contacts, thus mutants are unlikely to increase the chances of crystallizing a particular complex. However, it is possible to design mutants that can bind at only one site by making use of the crystal structures obtained so far. Such mutants will have a free surface that can participate in crystal contacts and that can be modified to improve its crystal contact-forming properties. Here, a comparison of two singlesite mutants that differ at three different positions is reported. In both mutants two different tryptophan residues participate in crystal-packing interactions, suggesting that this residue may be particularly interesting for enhancing crystalcontact formation.

\section{Introduction}

Several Gram-positive bacterial proteins interact with immunoglobulins. The best studied are Staphylococcus aureus protein A (SpA; Langone, 1982), streptococcal protein G (SpG; Björck \& Kronvall, 1984) and Peptostreptococcus magnus protein L (PpL; Björck, 1988). They are composed of two to five homologous repeats of immunoglobulin-binding domains that recognize distinct regions on a wide repertoire of immunoglobulins. Single domains of these immunoglobulin-binding proteins (IBP), PpL, SpA and SpG, can aid the crystallization of antibodies and their complexes because they provide alternative ways of forming a crystal lattice and thus they modify the chances of crystallizing the Fab and/or its complex (Stura et al., 2001; Stura, Graille et al., 2002). While, SpA and SpG domains recognize a common site at the interface between the $\mathrm{C}_{\mathrm{H}} 2$ and $\mathrm{C}_{\mathrm{H}} 3$ domains on the Fc of IgG (Fc $\gamma$; Deisenhofer, 1981; Sauer-Eriksson et al., 1995) and can thus be used in the crystallization of Fc-fusion proteins (Stura, Taussig et al., 2002) PpL does not. However, PpL can be used for the crystallization of Fabs such as SpG and SpA, each providing an interaction with a functionally distinct region on the Fab portion of particular immunoglobulin families or subclasses (Graille et al., 2001; Derrick \& Wigley, 1992; Graille et al., 2000). SpG binds to the $\mathrm{C}_{\mathrm{H}} 1$ domain and hence does not interact with $\mathrm{Fv}$ fragment, the smallest immunoglobulin portion that can bind antigen, nor with single-chain antibodies ( $\mathrm{scFv}$ ). A domain from streptococcal protein $\mathrm{G}$ has already been used to obtain crystals of a Fab-peptide complex (Derrick et al., 1999). SpA interacts with the $\mathrm{V}_{\mathrm{H}}$ domain on $12 \%$ of mouse and $50 \%$ of human Fv fragments. $\mathrm{PpL}$ is different from $\mathrm{SpA}$ and SpG as it binds with high affinity $\left(K_{\mathrm{d}}\right.$ of $\left.1 \mathrm{n} M\right)$ to the $\mathrm{V}_{\mathrm{L}}$ and not the $\mathrm{V}_{\mathrm{H}}$ domain. It is able to target up to $66 \%$ of mouse and $50 \%$ of human immunoglobulins. How $\mathrm{PpL}$ can bind tightly to a large population of immunoglobulin is explained by the crystal structure of the complex between a PpL single domain and a human Fab (Graille et al., 2001). In this structure the PpL domain is sandwiched between 
two antibody light chains. Only one of the two sites identified has already been documented (Wikström et al., 1995; Beckingham et al., 1999, 2001). The importance and the role of the second site has only been described recently (Housden et al., 2004).

The first single-site mutant was designed with a substitution from Asp55 to Ala (PpL-D55A; PDB code 1mhh) to disrupt an important salt bridge and a hydrogen bond crucial for the interaction through site 2. This mutant was built on the PpL-Y64W template where Trp64 replaces a tyrosine to provide an efficient fluorescent probe to measure $K_{\mathrm{d}}$ by stopped-flow methods. The crystal structure of the complex between the PpL-D55A-Y64W mutant and mouse Fab' 19D9D6 bearing a $\mathrm{V}_{\mathrm{L}} \mathrm{k} 9$ region to $2.1 \AA$ resolution. This crystallographic model confirmed the prediction of a single binding site (Graille et al., 2002). Here we report the crystallization and the crystal structure of the PpL-A66W mutant where Ala66 is mutated to Trp as an alternative fluorescent probe to Trp64 to measure $K_{\mathrm{d}}$ by stoppedflow methods. The results obtained with Trp66 and Trp64 are very different from each other (Housden et al., 2004) despite their proximity. The crystal structure of this mutant, also bound to Fab' 19D9D6, explains the difference in the behaviour between these two mutants and highlights the importance of the position of surface residues and in particular solvent-exposed tryptophans in the formation of crystal contacts.

\section{Materials and methods}

\subsection{Preparation of Fab' 19D9D6}

Murine monoclonal antibody 19D9D6 (IgG1, $\mathrm{V}_{\mathrm{L}} \mathrm{k}$; ; JolivetReynaud et al., 1998) was cultured, sequenced, expressed, cleaved and purified as described in detail elsewhere (Menez et al., 2003). Briefly, the IgG was purified on a protein A column and then cleaved with pepsin to yield $\mathrm{F}\left(\mathrm{ab}^{\prime}\right)_{2}$. The $\mathrm{F}\left(\mathrm{ab}^{\prime}\right)_{2}$ were purified by sizeexclusion chromatography and reduced to $\mathrm{Fab}^{\prime}$ fragments with mercaptoethanol followed by the blocking of the free cysteines with iodoacetamide. The $\mathrm{Fab}^{\prime}$ was then purified by size-exclusion chromatography.

\subsection{Mutagenesis and purification of the PpL mutants}

Mutant D55A-Y64W was made starting from the Y64W template DNA (Beckingham et al., 2001) while the A66W mutant was made from wild type. The tryptophan mutations were made to analyze the properties of the mutants in solution by stopped-flow fluorescence. The mutants discussed here, D55A-Y64W and A66W, were expressed in Escherichia coli as previously described (Beckingham et al., 2001) and stored in saturated ammonium sulfate at $30 \mathrm{mg} \mathrm{ml}^{-1}$ at $277 \mathrm{~K}$.

\subsection{Crystallization}

Complexes were prepared in solution before the crystallization trials, by mixing $18 \mu \mathrm{l}$ of Fab' 19D9D6 $\left(6 \mathrm{mg} \mathrm{ml}^{-1}\right)$ with $4 \mu \mathrm{l}$ of PpL mutant $\left(16 \mathrm{mg} \mathrm{ml}^{-1}\right)$. PpL stored at $32 \mathrm{mg} \mathrm{ml}^{-1}$ as an ammonium sulfate precipitate was diluted tenfold and re-concentrated three times in $0.05 \%$ sodium azide to reduce the salt present. Screening for crystallization was carried out using sitting-drop vapor diffusion at $290 \mathrm{~K}$ in an air-conditioned room, using three working solutions based on crystallization conditions for other Fab. These solutions were used in the screening and to grow crystals of the various Fab' 19D9D6 complexes: WS1 [24\%(w/w) monomethyl polyethylene glycol (MPEG 5000), $500 \mathrm{~m} M$ sodium chloride, $200 \mathrm{~m} M$ Tris- $\mathrm{HCl} \mathrm{pH}$ 9.0], WS2 [14\%(w/w) MPEG 2000, 10\% ethanol, $200 \mathrm{mM}$ imidazole malate $\mathrm{pH} 7.0$ ] and WS3 [10\%(w/w) MPEG 5000, $100 \mathrm{~m} M$ sodium acetate $\mathrm{pH} 4.5$ ]. Solution WS1 has been particularly successful in the
Table 1

Crystallization, data collection and refinement statistics for the data collected from crystals of the D55A-Y64W and A66W protein L mutant-Fab' 19D9D6 complexes.

\begin{tabular}{|c|c|c|}
\hline Data set & D55A-Y64W & A66W \\
\hline PDB code & $1 \mathrm{mhh}$ & $1 \mathrm{ymh}$ \\
\hline Crystallization & $\begin{array}{c}9 \% \text { MPEG } 5000,100 \mathrm{~m} M \\
\text { sodium acetate } \mathrm{pH} 4.5\end{array}$ & $\begin{array}{l}9 \% \text { MPEG } 5000,100 \mathrm{~m} M \\
\quad \text { sodium cacodylate } \mathrm{pH} 5.1\end{array}$ \\
\hline Drops (protein/reservoir) & $2.4 / 1.8$ & $1.5 / 1.5$ \\
\hline Cross-seeding & Yes (free Fab) & Spontaneous nucleation \\
\hline Data collection & ESRF-ID14EH1 & ESRF-ID29 \\
\hline Space group & $P 2_{1} 2_{1} 2_{1}$ & $P 2_{1} 2_{1} 2_{1}$ \\
\hline Unit-cell parameters $(\AA)$ & $\begin{array}{l}a=78.5, b=101.0 \\
\quad c=149.1\end{array}$ & $\begin{array}{l}a=77.143, b=111.47, \\
\quad c=148.70\end{array}$ \\
\hline Resolution range $(\AA)$ & $20.0-2.1$ & $20.0-2.6$ \\
\hline Completeness (\%) & 97.6 & 97.8 \\
\hline Multiplicity & 6.77 & 10.0 \\
\hline$\langle I / \sigma(I)\rangle$ & 26.6 & 21.0 \\
\hline$R_{\text {sym }}(\%)$ & 6.7 & 5.8 \\
\hline Refinement $\dagger$ & $C N S$ & CCP4 \\
\hline$R_{\text {free }}(\%)$ & 24.7 & 31.0 \\
\hline$R_{\text {work }}(\%)$ & 19.7 & 23.1 \\
\hline
\end{tabular}

$\dagger$ CNS (Brünger et al., 1998), CCP4 (REFMAC; Murshudov et al., 1997).

crystallization of various Fabs and is related to that used in the crystallization of Fab 2A2 in complex with wild-type PpL (13-16\% MPEG 5,000, $100 \mathrm{~m} M$ imidazole malate $\mathrm{pH}$ 8.5; Graille et al., 2001) and to the solution used for Fab 4x11 [16\%(w/w) MPEG 2000, $50 \mathrm{mM}$ sodium borate pH 9.0; Stura, Tête-Favier et al., 2002]. Small needles were obtained with this solution. Larger more promising needles were obtained with WS3, the solution used for the crystallization of the complex between the single site binding PpL mutant D55AY64W and Fab' 19D9D6 (Graille et al., 2002). This suggested that the crystal form might be identical or very similar to the those of the PpLD55A-Y64W mutant. However, in order to obtain large single crystals, the $\mathrm{pH}$ had to be increased to 5.1. The final working solution was $10 \%$ MPEG 5000, $100 \mathrm{~m} M$ sodium cacodylate $\mathrm{pH}$ 5.1. The sitting drops consisted of $1.5 \mu \mathrm{l} \mathrm{Fab-PpL}$ complex and the same volume of reservoir solution which was placed on the protein drop without mixing. A range from $100 \%$ working solution to $84 \%$ was used to grow crystals. More than one crystal per drop was obtained in the range $100-90 \%$ and a single crystal was obtained in the range $88-84 \%$ but after one month and six months respectively, probably only after evaporation through the vacuum grease.

\subsection{Data collection}

Data were collected from crystals transferred, as described in Stura \& Gleichmann (1999), into a cryoprotectant solution containing $5 \mu \mathrm{l}$ ethylene glycol, $3 \mu \mathrm{l} \mathrm{50 \% (w/w)} \mathrm{MPEG} 5000$ and $10 \mu \mathrm{l} 10 \%(w / w)$ MPEG 5000, $3 \mathrm{mM} \mathrm{ZnCl} 2,3 \mathrm{~m} M \mathrm{CdCl}_{2}, 100 \mathrm{~m} M$ sodium cacodylate pH 6.5. This solution is not optimal and many crystals crack upon transfer. Other solutions tested produce worse results. We were able to collect data from a single crystal that was left in the cryogenic solution only briefly, before cryocooling the crystal in a loop in liquid ethane. Since the data obtained at the ESRF synchrotron facility (Grenoble, France) on beamline ID29 extended to $2.6 \AA$. A similar difficulty was experienced also for Fab' 19D9D6-PpL-D55A complex crystals, where data were first recorded at $100 \mathrm{~K}$ to $2.6 \AA$ on beamline ID14-EH1 and later, after improvements in crystal quality, to $2.1 \AA$ resolution on ID14-EH4 at the ESRF synchrotron facility (Grenoble, France).

The severity of the cryosoaking problem appears to be worse for the PpL-A66W complex and future improvements may include growing crystals under cryogenic compatible crystallization conditions instead of soaking the crystals in a cryosolution. Better data 


\section{conference papers}

quality may be achieved in this way. The data were processed using the HKL package (Otwinowski \& Minor, 1997). These crystals belong to the orthorhombic space group $P 22_{1} 2_{1} 2_{1}$, with unit-cell parameters $a=77.143, b=111.47, c=148.70 \AA$.

\subsection{Structure determination}

The structure was solved by molecular replacement using MOLREP (CCP4; Vagin \& Teplyakov, 1997) with a single Fab-PpL complex from the Fab' 19D9D6-PpL-D55A-Y64W complex (PDB code $1 \mathrm{mhh})$. The molecular replacement located correctly the two $\mathrm{Fab}^{\prime}$ complexes in the asymmetric unit. The choice of asymmetric unit for PpL-A66W is different from that used in $1 \mathrm{mhh}$ as such a choice is not compatible with the PpL-A66W lattice and all attempts at obtaining a molecular-replacement solution with the two complexes in the $1 \mathrm{mhh}$ asymmetric unit failed. The programs in the XtalView

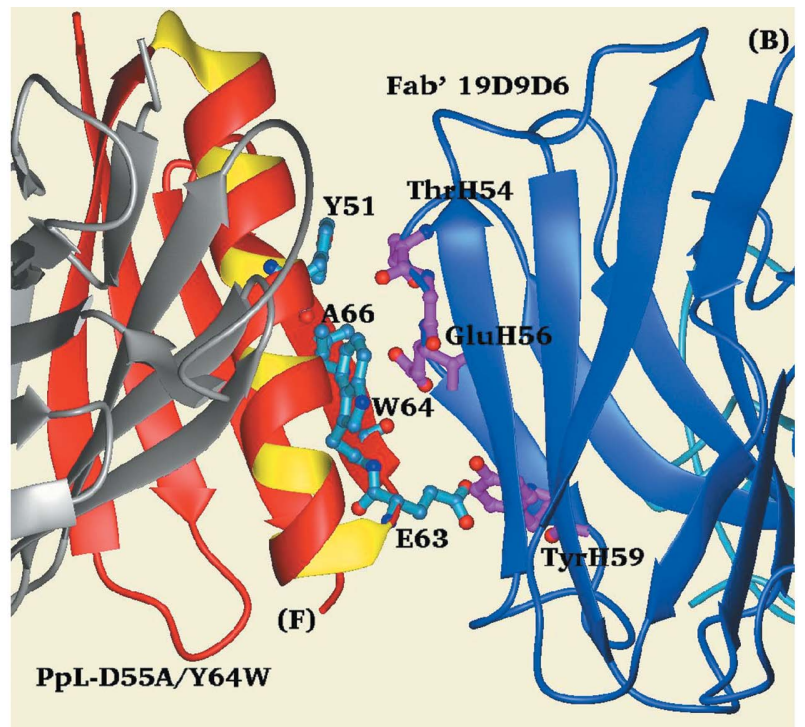

(a)

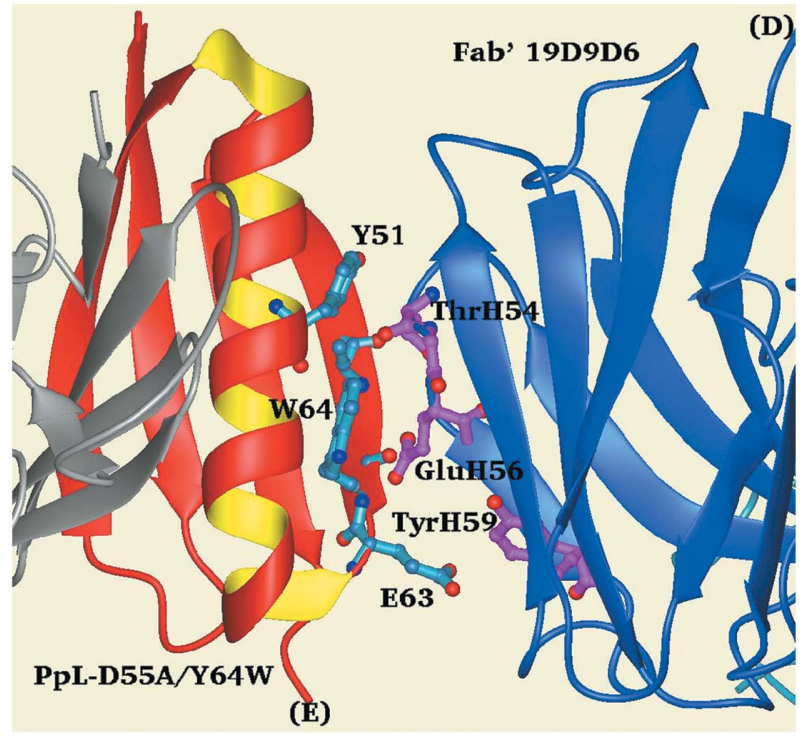

(b) suite (McRee, 1999) were used for electron-density map interpretation and other graphical analysis. Refinement was carried out with REFMAC from the CCP4 suite of programs. $R_{\text {free }}$ and $R_{\text {work }}$ are 31.0 and $23.1 \%$, respectively (Table 1 ).

\section{Results}

\subsection{Overall packing}

The crystal habit, space group, $P 2_{1} 2_{1} 2_{1}$, and unit-cell parameters for PpL-A66W are reminiscent of those for the complex between Fab 19D9D6 and the single site-binding PpL mutant D55A-Y64W with unit-cell parameters $a=78.5, b=101.0, c=149.1 \AA$. The largest deviation is noted for the $b$ direction which is $10 \AA$ longer ( $b=111.47 \AA$ for the PpL-A66W complex). For both PpL-D55AY64W and PpL-A66W there are two complexes in the asymmetric unit and each complex consists of one Fab' 19D9D6 and one PpL mutant. Thus, as in the PpL-D55A-Y64W complex there is no lightchain binding at site 2 (Graille et al., 2002). This observation is consistent with stopped-flow fluorescence analysis (Housden et al., 2004).

The packing of the complexes in the unit cell is similar despite the shift in the $b$ direction and the complexes in the PpL-A66W lattice roughly superimpose on those for the PpL-D55A-Y64W complex. The two complexes in the asymmetric unit of the PpL-A66W have been selected to have the highest intermolecular contact area and form a dimer around $\mathrm{IleH} 2$ from the Fab heavy chain. The same dimeric arrangement is found in the PpL-D55A-Y64W lattice.

\subsection{Crystal contacts}

Although overall the packing is similar, there are important differences within the contacts. In the PpL-D55A-Y64W lattice, Trp64 mediates the two major crystal contacts made by $\mathrm{PpL}$ with residues H53-H58 of the Fab heavy chain. The two contacts are not crystallographically equal but they are almost identical. There is no obvious stereochemical reason why the two crystal contacts could not accommodate the mutations, A66W and W64Y. However, when these

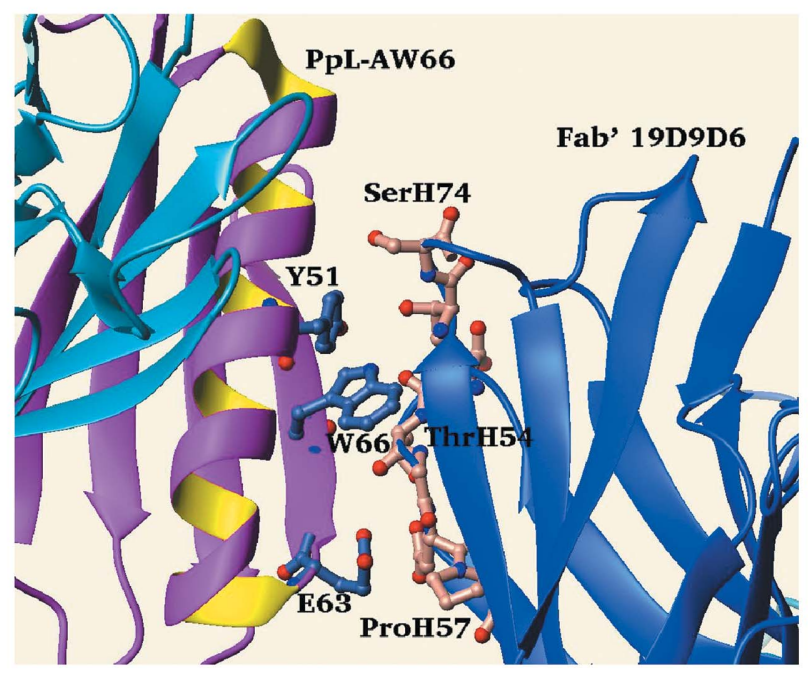

(c)

Figure 1

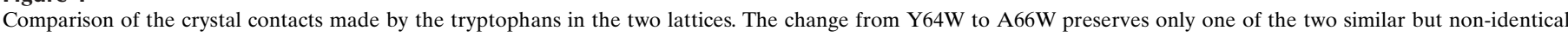
contacts found in the D55A-Y64W structure (left). Although this contact is replaced by another one that is very similar, the minor differences are magnified at long distances leading to a $10 \AA$ shift in the $b$ direction (approximately vertical in the figures). This is reflected by the shift in position of ThrH54. 
residues are varied, the contacts are modified and a strong asymmetry is created between the contacts. One of the Trp66 is at the center of an expanded patch of interactions. The increase in size has been achieved by recruiting the strand H69-H74 adjacent to residues H53H57 of the Fab heavy chain, which includes LeuH71, with which the tryptophan interacts directly. On the other hand, the equivalent crystal contact is reduced in scope. It still includes residues $\mathrm{H} 53-\mathrm{H} 57$ but now Trp66 retains just one van der Waals interaction with a proline and in this patch of interactions it is no longer a dominant residue and it does not become fully shielded from solvent as a result of the interaction. Although locally the changes are relatively minor, they have large consequences at a distance. The contact between the two complexes found in the $1 \mathrm{mhh}$ asymmetric unit is broken as the molecules glide past each other and separate and this separation results in the increase in cell size by $10 \AA$ in the $b$ direction. Thus, the lattice formed by PpL-A66W is likely to be weaker than that formed by PpL-D55A-Y64W. This is reflected in the susceptibility of the crystals to even the shortest soak in cryosolvents. This intolerance to changes is even more accentuated than for the PpL-D55A-Y64W complex crystals.

\section{Discussion}

The two mutants PpL-A66W and PpL-D55A achieve the elimination of site 2 binding in distinct ways. In the case of PpL-A66W, single-site binding is achieved through steric hindrance at site 2 , where the large tryptophan side chain would clash with both ProL8 and LeuL11. In the case of PpL-D55A-Y64W the truncation of the Asp55 side chain sufficiently weakens site 2 binding by preventing the formation of a salt bridge between the aspartate side chain with the side chain of Arg or LysL24. Without the elimination of site 2, the small PpL is sandwiched between two immunoglobulins and cannot mediate crystal contacts so that engineering of its surface is unlikely to yield mutants with new and interesting properties for Fab complex crystallization. Thus both these mutants are site-1-only light-chain binders and either can be used as a platform for the creation of a large repertoire of single-site binders. By mutating surface residues (not involved in interactions at site-1) a large library of mutants to be used in the creation of contacts in crystals of murine and human antibodies (whole IgG, Fab and scFv) both free and antigen complexed.

The use of tryptophan for stopped-flow fluorescence studies appears to have a fortuitous parallel in crystallization. In both the mutants PpL-D55A-Y64W and PpL-A66W, these engineered tryptophans mediate crystal contacts. Given the nature of the residue it is not surprising to find that it mediates crystal contacts, but it was unexpected to discover that as the tryptophan is moved along the $\mathrm{PpL}$ sequence it is capable of inducing what turns out to be relatively large changes in packing. Locally the modifications are small, but they have the strength to alter other contacts which at first glance would appear of equal or greater importance. These observations indicate that crystal packing may be modified and controlled through mutation of surface residues and that a fine balance is at play between the various interaction patches that create the three-dimensional crystal lattice. The interactions between patches can be rather fluid and individual contacts between residues is not maintained even when the overall packing is relatively well conserved as are the unit-cell parameters (along the $a$ and $c$ directions). Here we suggest that mutations of exposed residues to tryptophan might have a high potential for the formation of crystal contacts compared with most other residues. Tryptophan is among those residues that establish favorable interactions with many other residues (Dasgupta et al., 1997) and in particular with lysine, a residue which is considered to be otherwise less prone to establish crystal contacts because of their intrinsic entropy and that its truncation to alanine can positively affect crystallizability (Longenecker et al., 2001).

In conclusion, we concur with Longenecker and coworkers that mutagenesis of surface residues can be a more effective approach for the crystallization of recalcitrant proteins than the extensive and often random screening of large numbers of different crystallization conditions. The two mutagenesis approaches follow opposite but complementary direction, the first consists of the truncation of highentropy side chains while we focus on the mutation or addition of residues chains capable of mediating interactions: cysteines (Stura, Tête-Favier et al., 2002) and tryptophans. All mutagenesis approaches expose themselves to the danger that by trying to augment the potential of the protein to form new interactions, the protein may also become more prone to non-specific aggregation, a problem that is often encountered as potential glycosylation sites are eliminated in this manner.

The problem remains that each mutated protein represents a single example that cannot be considered statistically significant. However, PpL single-site mutants have the advantages that they can be used complexed to a large repertoire of antibody and antibody complexes giving us the chance of following up the study over a larger ensemble.

We thank the staff at the European Synchrotron Radiation Facility (ESRF) in Grenoble on beamlines ID14-EH1, ID14-EH4 and ID29 and Marie-Hélene Le Du for help in collecting data.

\section{References}

Beckingham, J. A., Bottomley, S. P., Hinton, R., Sutton, B. J. \& Gore, M. G. (1999). Biochem. J. 340, 193-199.

Beckingham, J. A., Housden, N. G., Muir, N. M., Bottomley, S. P. \& Gore, M. G. (2001). Biochem. J. 353, 395-401.

Björck, L. (1988). J. Immunol. 140, 1194-1197.

Björck, L. \& Kronvall, G. (1984). J. Immunol. 133, 969-974.

Brünger, A. T., Adams, P. D., Clore, G. M., DeLano, W. L., Gros, P., GrosseKunstleve, R. W., Jiang, J.-S., Kuszewski, J., Nilges, M., Pannu, N. S., Read, R. J., Rice, L. M., Simonson, T. \& Warren, G. L. (1998). Acta Cryst. D54, 905-921.

Dasgupta, S., Iyer, G. H., Bryant, S. H., Lawrence, C. E. \& Bell, J. A. (1997). Proteins, 28, 494-514.

Deisenhofer, J. (1981). Biochemistry, 20, 2361-2370.

Derrick, J. P., Feavers, I. \& Maiden, M. C. (1999). Acta Cryst. D55, 314-316.

Derrick, J. P. \& Wigley, D. B. (1992). Nature (London), 359, 752-754.

Graille, M., Harrison, S., Crump, M. P., Findlow, S. C., Housden, N. G., Muller, B. H., Battail-Poirot, N., Sibaî, G., Sutton, B. J., Taussig, M. J., JolivetReynaud, C., Gore, M. G. \& Stura, E. A. (2002). Biol. Chem. 277, 4750047506.

Graille, M., Stura, E. A., Housden, N. G., Beckingham, J. A., Bottomley, S. P., Beale, D., Taussig, M. J., Sutton, B. J., Gore, M. G. \& Charbonnier, J.-B. (2001). Structure, 9, 679-687.

Graille, M., Stura, E. A., Taussig, M. J., Corper, A., Sutton, B. J., Charbonnier, J.-B. \& Silverman, G. J. (2000). Proc. Natl Acad. Sci. USA, 97, 5399-5404.

Housden, N. G., Harrison, S., Housden, H. R., Thomas, K. A., Beckingham, J. A., Roberts, S. E., Bottomley, S. P., Graille, M., Stura, E. A. \& Gore, M. G. (2004). J. Biol. Chem. 279, 9370-9378.

Jolivet-Reynaud, C., Dalbon, P., Viola, F., Yvon, S., Paranhos-Baccala, G., Piga, N., Bridon, L., Trabaud, M. A., Battail, N., Sibai, G. \& Jolivet, M. (1998). J. Med. Virol. 56, 300-309.

Langone, J. J. (1982). Adv. Immunol. 32, 157-252.

Longenecker, K. L., Garrard, S. M., Sheffield, P. J. \& Derewenda, Z. S. (2001). Acta Cryst. D57, 679-688.

McRee, D. E. (1999). J. Struct. Biol. 125, 156-165.

Menez, R., Bossus, M., Muller, B. H., Sibai, G., Dalbon, P., Ducancel, F., Jolivet-Reynaud, C. \& Stura, E. A. (2003). J. Immunol. 170, $1917-$ 1924.

Murshudov, G. N., Vagin, A. A. \& Dodson, E. J. (1997). Acta Cryst. D53, 240 255. 


\section{conference papers}

Otwinowski, Z. \& Minor, W. (1997). Methods Enzymol. 276, 307326.

Sauer-Eriksson, A. E., Kleywegt, G. J., Uhlen, M. \& Jones, T. A. (1995). Structure, 15, 265-278.

Stura, E. A. \& Gleichmann, T. (1999). Crystallization of Nucleic Acids and Proteins, a Practical Approach, 2nd ed., edited by A. Ducruix \& R. Giegé, pp. 365-390. Oxford University Press.

Stura, E. A., Graille, M. J. \& Charbonnier, J.-B. (2001). J. Cryst. Growth, 232, 573-579.
Stura, E. A, Graille, M., Housden, N. G. \& Gore, M. G. (2002). Acta Cryst. D58, 1744-1748.

Stura, E. A., Taussig, M. J., Sutton, B. J., Duquerroy, S., Bressanelli, S., Minson, A. C. \& Rey, F. A. (2002). Acta Cryst. D58, 1715-1721.

Stura, E. A., Tête-Favier, F., Muller, S. \& Aubry, A. (2002). Acta Cryst. D58, $1740-1743$.

Vagin, A. \& Teplyakov, A. (1997). J. Appl. Cryst. 30, 1022-1025.

Wikström, M., Sjöbring, U., Drakenberg, T., Forsén, S. \& Björck, L. (1995). J. Mol. Biol. 250, 128-133. 